\title{
Neospora caninum Infection in a Litter of Labrador Retriever Dogs in Denmark
}

Neospora caninum is a recently recognized cyst-forming coccidian parasite associated with severe encephalomyelitis and myositis in dogs of different breeds and ages (Bjerkås et al. 1984, Bjerkås \& Presthus 1988, Dubey et al. 1988), but has for many years been misdiagnosed as Toxoplasma gondii. In some dogs, the main clinical sign has been attributed to polyradiculoneuritis (Dubey et al. 1988, Cuddon et al. 1992). Furthermore, ulcerative dermatitis (Dubey et al. 1988) and megaoesophagus have been reported (Wolf et al. 1991). The life cycle of the parasite and mode of infection have not been clarified, but transplacental infection seems so far to be the natural route of transmission between intermediate hosts (Dubey \& Lindsay 1989). It has been speculated that the disease in young and adult dogs might be due to reactivation of a persistent infection because corticosteroid therapy can activate a latent $N$. caninum infection (Dubey \& Lindsay 1993).

Since the infection (neosporosis) was first recognized in Norway (Bjerkås et al. 1984) and a similar parasite was identified in USA and named Neospora caninum (Dubey et al. 1988), the infection has been diagnosed in Scandinavia, several other European countries, Australia, South Africa, and Japan (Dubey \& Lindsay 1993). The infection is found sporadically, and only limited information on the prevalence of the infection is available. Examination of the prevalence of subclinical infection in a population of urban dogs in England showed that 27 of 163 dogs (16.6\%) had anti- bodies against $N$. caninum (Trees et al. 1993), and in USA, $2.2 \%$ were positive out of 229 dogs examined (Lindsay et al. 1990). Neosporosis has also been described in cattle, sheep, horses, and goats (Dubey \& Lindsay 1993). In the present paper, $N$. caninum infection in a Labrador Retriever litter diagnosed in Denmark in 1993 is described.

The litter consisted of 8 Labrador Retriever dogs. One male dog was, without being examined by us, euthanised at 12 weeks of age due to exercise intolerance, weakness, ataxia, and muscle atrophy. Another dog from the bitch's litter 2 years earlier had shown similar clinical signs. The remaining 7 puppies, 2 males and 5 females, and the bitch were evaluated clinically and serologically for antibodies to $N$. caninum. At 12 weeks of age, 1 female puppy, which had showed exercise intolerance and ataxia for about 4 weeks, was admitted to the Small Animal Hospital of the Department of Clinical Studies, The Royal Veterinary and Agricultural University, Copenhagen. It was observed for 3 weeks before euthanasia. This case is described in detail here. The mother and the other 6 puppies were given a physical examination, and pelvic radiographs were obtained in 1 of the puppies at an age of 6 months. The clinical examination of the admitted dog included neurological examination, electrocardiography, radiological examination, biochemical, and haematological tests.

The mother and the 6 puppies were examinated by physical, and pelvic radiographs were 


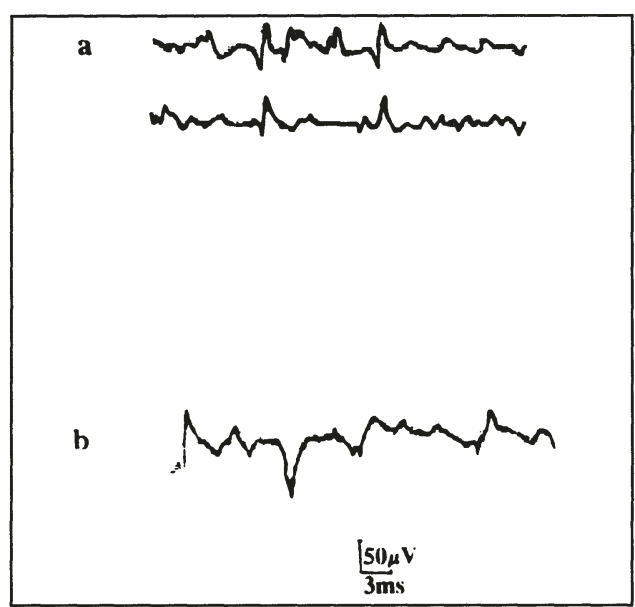

Figure 1. Concentric needle EMG. Spontaneous activity with fibrillation potentials (a) and positive sharp waves (b).

obtained in 1 of the puppies at an age of 6 months.

After walking for about $5 \mathrm{~min}$, the hospitalized dog showed weakness of the hindlegs, it moved with stiff pelvic limbs with intermittent knuckling of the paw and occasional falling. The dog was running in a bunny-hopping manner. Slight muscle atrophy was observed in most muscles. Pelvic limb postural reactions, patellar tendon, and pedal reflexes were depressed. The dog was alert, mentally normal and interested in playing. The clinical signs gradually detoriorated. The generalized muscle atrophy became marked. Both stifles and tarsal joints became hyperextended with restriction of joint movement. Patellar tendon reflexes were lost, and the pedal reflexes markedly depressed. The dog was able to stand unassisted. It walked with stiff stifles and tarsal joints, but was easily fatigued. The mental condition, temperature, and appetite were normal throughout the observation period.

The results of routine haematological examinations were within normal limits. An in- crease ( 5 times normal) in serum creatinine kinase was detected. The electrocardiogram was normal. The hips and spine were radiographically normal.

One further puppy from the litter showed a stiff pelvic limbs gait from the age of 16 weeks without radiographic evidence of dysplasia. No progression of the clinical signs was seen during an observation period of 6 months. The bitch and the remaining 5 puppies showed no abnormal clinical signs at any time. On the hospitalized dog, electrophysiological tests were performed under anaesthesia induced with intravenous thiopentane and maintained by halothane in nitrous oxide and oxygen. An . electromyographic examination using a concentric electrode was performed on the left thoracic and left pelvic limb. Motor and sensory nerve conduction velocities were measured in the left sciatic and tibial nerve. Repetitive stimulation of the sciatic nerve and of the ulnar nerve was performed at 3,15 , and $50 \mathrm{~Hz}$ in $20 \mathrm{sec}$ followed by $3 \mathrm{~Hz}$ in $3 \mathrm{sec}$, and $15 \mathrm{~Hz}$ in $5 \mathrm{~min}$ followed by $3 \mathrm{~Hz}$ in $3 \mathrm{sec}$ (Flagstad et al. 1989). Neostigmine $(0.5 \mathrm{mg} / 10 \mathrm{~kg}$ body weight) was given intravenously before and during the $15 \mathrm{~Hz}$ repetitive stimulation test. Denervation potentials including fibrillation potentials and positive sharp waves were found primarily in the pelvic limb (Fig. 1). Fibrillation potentials and positive sharp waves were also found, but more localized in the thoracic limb muscles, in the musculus temporalis, and in musculus pterygoideus. The compound muscle action potential amplitude was 1-4 mV after sciatic, tibial or ulnar nerve stimulation. Motor nerve conduction velocity of the sciatic/tibial nerve was $44-48$ $\mathrm{m} / \mathrm{s}$, and the sensory nerve conduction velocity of the tibial nerve was $40 \mathrm{~m} / \mathrm{s}$, with an evoked potential amplitude of $0.36 \mu \mathrm{V}$. Repetitive stimulation of the sciatic nerve with $3 \mathrm{~Hz}$ in $3 \mathrm{sec}$ revealed a decrease of $10-13 \%$. Stim- 


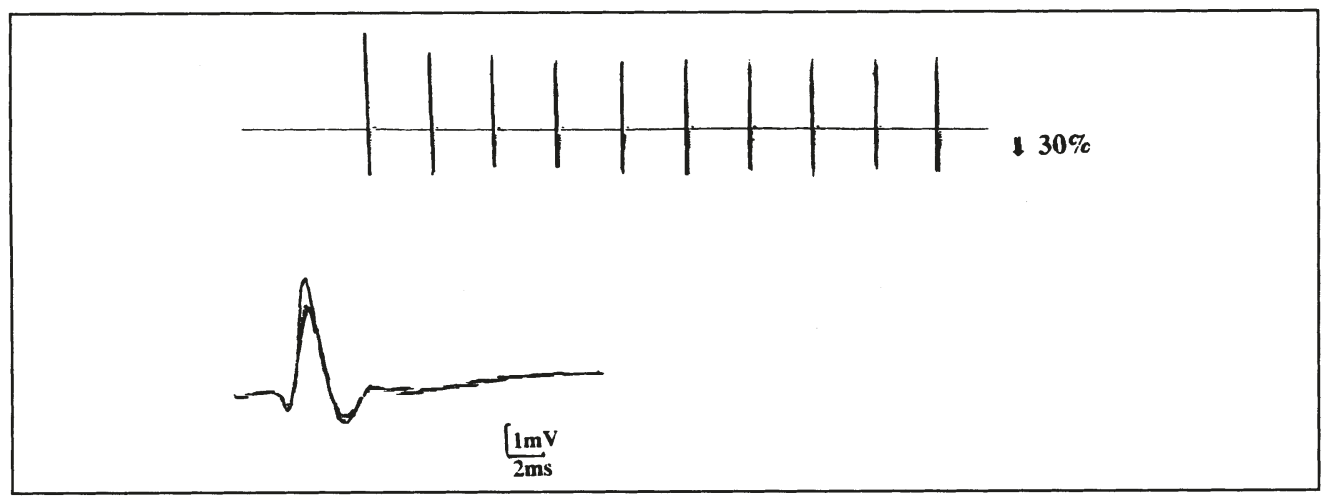

Figure 2. Repetitive nerve stimulation at $3 \mathrm{~Hz}$ for $3 \mathrm{sec}$. Decremental response of the biceps femoris muscle after stimulation of the sciatic nerve. A decrease of $30 \%$ of the muscle action potential is seen.

ulation with $15 \mathrm{~Hz}$ for $20 \mathrm{sec}$ and $50 \mathrm{~Hz}$ for 20 sec caused a gradual amplitude decrease of up to $70 \%$. The action potential recovered immediately when single stimuli were given 2,5 , and $10 \mathrm{sec}$ after tetanic stimulation. The subsequent $3 \mathrm{~Hz}$ stimulation revealed a decrement of up to $40 \%$ (Fig. 2). On exposure to 15 $\mathrm{Hz}$ for $5 \mathrm{~min}$, there was a gradual decrease in amplitude of $80 \%$, followed by a decrement of up to $64 \%$ at stimulation at $3 \mathrm{~Hz}$ for $3 \mathrm{sec}$. The decrement at $3 \mathrm{~Hz}$ persisted for $20 \mathrm{~min}$ or longer. Injection of neostigmine before and during $15 \mathrm{~Hz}$ stimulation could not prevent the "electrical fatigue" or the decrement at 3 $\mathrm{Hz}$ stimulation. Repetitive stimulation of the ulnar nerve provoked about $40 \%$ decrement at $15 \mathrm{~Hz}$ stimulation followed by $30 \%$ decrease at $3 \mathrm{~Hz}$ stimulation for $3 \mathrm{sec}$.

Sera from the 7 puppies and their mother were examined. One puppy (the hospitalized case described in this paper) was 12 weeks, and the remaining 6 were 6 months old at the time of testing. The sera were tested at dilutions of 1:200 and 1:600 as described elsewhere (Bjekås \& Presthus 1988), and all showed a positive reaction to the $N$. caninum antigen at the highest dilution.

The hospitalized dog was euthanised with pentobarbital, and a complete necropsy was performed. Tissue specimens for histological and immunohistochemical studies were taken from the right sciatic nerve, the central nervous system (cerebrum, cerebellum, medulla oblongata, and spinal cord), and muscles from the right pelvic limb (semitendinosus, biceps femoris, quadriceps femoris, and tibialis cranialis). Tissues were fixed in $10 \%$ neutral buffered formalin, dehydrated and embedded in paraffin. Sections of $5 \mu \mathrm{m}$ were cut and then stained with haematoxylin and eosin (HE), periodic acid-Schiff (PAS), and by the Grocott's methenamine silver (GMS) method. Specific immunostaining of N. caninum and T. gondii organisms was done as described by Bjekås \& Presthus 1988.

Gross lesions were restricted to the skeletal muscles, especially of the limbs, which were pale, moist, and atrophic. Microscopically, cerebral and cerebellar parasitic cysts containing organisms similar to bradyzoites of $N$. caninum were seen (Bjerkås \& Presthus 1988). The parasites were often associated with focal encephalitis characterized by gliosis, malacia, vasculitis, and formations of perivascular cuffs. Mononuclear leptomeningitis was seen focally. In medulla oblongata and the spinal 
cord, focal gliosis was present. No lesions of the sciatic nerve was observed. Necrosis, vasculitis, and infiltration of mononuclear cells, including plasma cells and lymphocytes, were widespread in all muscles, and groups of parasitic organisms resembling tachyzoites of $N$. caninum were regularly found within muscle fibres. Immunohistochemically, the identity of the parasites as being $N$. caninum was confirmed by staining with specific antiserum against $N$. caninum, whereas no staining was obtained with the antiserum against $T$. gondii. The finding of antibodies to N. caninum in the bitch and all the 7 puppies suggests a transplacental or colostral transmission of the infection. This view is in accordance with the fact that transplacental transmission in dogs also can be established experimentally (Dubey \& Lindsay 1989).

Based on the results of the physical and neurological examinations, a progressive generalized neuromuscular disorder was suspected. The differential diagnosis included a congenital or metabolic myopathy; polymyositis of various etiology, viral infection, protozoal infection (toxoplasmosis and neosporosis), or immune-mediated disease; a neuromuscular junction disorder, in particular a congenital myasthenia gravis syndrome; and a polyneuropathy due to congenital, inflammatory, metabolic, or toxic causes.

The most prominent clinical sign was exercise intolerance, electrophysiologically corresponding to the finding of a neuromuscular block characterized by a progressive decrement of muscle action potentials evoked by repetitive motor nerve stimulation. The electromyographical finding of spontaneous activity with fibrillation and positive sharp wave found in some of the muscles is characteristic of a neurogenic disorder. The motor nerve conduction velocity was, however, within normal limits for the age (Sims \& Redding 1980), which would be expected to be decreased in a neurogenic disorder, i.e. polyneuropathy (Wolf et al. 1991, Cuddon et al 1992).

The amplitude of the evoked muscle action potential was lower, 1- $4 \mathrm{mV}$, than expected for the age (Sims \& Redding 1980). A low evoked muscle action potential amplitude is especially found in myopathic disorders ( $\mathrm{Ki}$ mura 1983) i.e. a myopathy or a polymyositis. A low action potential amplitude is also characteristic of neuromuscular junction disorders of presynaptic origin (Oh 1992), which were consistent with the finding of a lack of response to neostigmine in the repetitive stimulation test.

The etiological diagnosis was confirmed at the post mortem examination by immunohistochemical staining of $N$. caninum organisms in connection with widespread polymyositis and encephalomeningomyelitis as described by Bjerkås \& Presthus (1988) and by Dubey et al (1988). The exercise intolerance, the progressive nature of the disease, and the elevated creatine kinase in serum is consistent with a polymyositis condition with necrosis of muscle fibres. The spontaneous activity can be explained by the metabolic changes due to necrosis of the fibres. Similarly, the neuromuscular block found by repetitive stimulation may be explained by the destruction of many muscle fibres and thus being a small reserve of muscle fibres available for force generation.

The final diagnosis might have been established clinically by the examination of muscle biopsies. The characteristic feature of polymyositis consisting of necrosis of individual fibres together with inflammatory cell infiltrates is instantly recognizable, but as the number of parasites may vary and be missing in the individual sample (Cuddon et al. 1992), most cases have been diagnosed post mortem. We have for the first time described a case of 
canine neosporosis in Denmark, and based on observations in the present case it can be concluded that clinical, electrophysiological, and serological examinations, although suggestive of a neuromuscular disorder, should be accomplished by immunohistopathological examination in order to state a final diagnosis of neosporosis in dogs.

\section{A. Flagstad ${ }^{1}$, H. E. Jensen ${ }^{1}$, I. Bjerkås ${ }^{2}$ and K. Ras- mussen ${ }^{3}$. \\ ${ }^{1}$ Departments of Clinical Studies and of Pharmacol- ogy and Pathobiology, The Royal Veterinary and Agricultural University, Frederiksberg, Denmark, ${ }^{2}$ Department of Morphology, Genetics and Aquatic Biology, Norwegian College of Veterinary Medicine, Oslo, Norway, and ${ }^{3}$ West Lolland Small Animal Clinic, Denmark.}

\section{References}

Bjerkås I, Mohn SF, Presthus J: Unidentified cystforming sporozoon causing encephalomyelitis and myositis in dogs. Z. Parasitenkd. 1984, 70, 271-274.

Bjerkås I, Presthus J: Immuno-histochemical and ultrastructural characteristics of a cystforming sporozoon associated with encephalomyelitis and myositis in dogs. APMIS. 1988, 96, 445-454.

Cuddon $P$, Lin D-S, Bowman DD, Lindsay DS, Miller TK, Duncan JD, deLahunta A, Cummings J, Suter M, Cooper B, King JM, Dubey JP: Neospora Caninum infection in English Springer Spaniel littermates. Diagnostic evaluation and organism isolation. J. vet. intern. Med. 1992, 6, 325-332.
Dubey JP, Carpenter JL, Speer CA, Topper MJ, Uggla $A$ : Newly recognized fatal protozoan disease of dogs. J. Amer. vet. med. Ass. 1988, 192, 12691285.

Dubey JP, Lindsay DS: Transplacental Neospora caninum infection in dogs. J. Amer. vet. Res. 1989, 50, 1578-1579.

Dubey JP, Lindsay DS: Neosporosis. Parasitol Today. 1993, 9, 452-458.

Flagstad A, Trojaborg W, Gammeltoft S: Congenital myasthenic syndrome in the dog breed Gammel Dansk Hønsehund: Clinical, electrophysiological, pharmacological and im munological comparison with acquired myasthenia gravis. Acta vet. scand. 1989, 30, 89-102.

Kimura J: Electrodiagnosis in diseases of nerve and muscle. 2nd ed, F.A. Davies, Philadelphia. 1983.

Lindsay DS, Dubey JP, Upton SJ, Ridley RK: Serological prevalence of Neospora caninum and Toxoplasma gondii in dogs from Kansas. J. helminthol. Soc. $1990,57,86-88$.

Oh SJ: Repetitive nerve stimulation test. Meth. clin. Neurophys. 1992, 3, 1-15.

Sims $M H$, Redding $R W$ : Maturation of nerve conduction velocity and the evoked muscle potential in the dog. J. Amer. vet. Res. 1980, 41, 1247-1251.

Trees AJ, Guy F, Tennant BJ, Balfour AH, Dubey JP: Prevalence of antibodies to Neospora caninum in a population of urban dogs in England. Vet. Rec. 1993, 132, 125-126.

Wolf M, Cachin M, Vandevelde M, Tipold A, Dubey JP: Zur klinischen Diagnostic des Protozoären Myositissyndroms (Neospora caninum) des Welpen (Clinical diagnosis of protozoal myositis-encephalitis syndrome (Neospaa caninum) in puppies.) Tierärztl. Prax. 1991, 19, 302-306.

(Received January 5, 1995; accepted May 8, 1995).

Reprints may be requested from: A. Flagstad, Department of Clinical Studies, The Royal Veterinary and Agricultural University, Bülowsvej 13, DK-1870, Frederiksberg C, Denmark. 


